cambridge.org/cty

\section{Letter to the Editor}

Cite this article: Li A, Guo Y, and Yao G (2019) Valuable research for recognising the relationship between the autonomic nervous system disturbance and migraine. Cardiology in the Young 29: 1120-1120.

doi: $10.1017 /$ S1047951119001574

Received: 23 April 2019

Accepted: 31 May 2019

First published online: 9 July 2019

\section{Author for correspondence:}

G. Yao, Department of Neurology, the Second Hospital of Jilin University, Changchun 130041, Jilin, China. Tel: + 860431 81136298; Fax: + 86 0431 81136298; E-mail: yaogang0431@163.com

\title{
Valuable research for recognising the relationship between the autonomic nervous system disturbance and migraine
}

\section{An-ran Li, Yu Guo and Gang Yao}

Department of Neurology, The Second Hospital of Jilin University, Changchun, Jilin, China

Dear Editor,

We read the recent paper by Tosun et $\mathrm{al}^{1}$ published in Cardiology in the Young with great interest because it focussed on investigating the Electrocardiogram (ECG) changes during attack-free period in children with migraine, in terms of QTc interval, QTc, and P-wave dispersion to evaluate the autonomic nervous system disturbance. In their study, children with migraine showed significantly lower duration of $\mathrm{P}$ minimum and significantly higher duration of $\mathrm{P}$ maximum and $\mathrm{P}$ dispersion compared with the control group. According to their findings, although migraine patients were asymptomatic and no arrhythmia was detected in the surface ECG, sympathovagal balance in the sympathetic system, which may be disrupted in favour of the sympathetic system, should continue even in the attack-free period. We, however, find it interesting that the specific targeted children with migraine in this study did not include clinical subtypes of migraine.

In 2015, Matei et $\mathrm{al}^{2}$ investigated 27 patients with migraine (10 with migraine with aura and 17 without aura) during headache-free periods and 10 age-matched healthy control patients. The results suggested that reduced parasympathetic activity with sympathetic predominance was found in migraine patients more pronounced during the night period; most affected were those with migraine with aura.

On the basis of our own experience, the relationship between the migraine and the autonomic nervous system disturbance is closely related to its clinical subtypes. ${ }^{3}$ Monroe et $\mathrm{al}^{4}$ reported a fatal case involving a young man with migraine-induced syncope and bradycardia with subsequent pacemaker implantation who died unexpectedly. According to the headache characteristics, we highly speculate that the migraine reported by Monroe is a rare type Migraine with brainstem aura. ${ }^{5}$ This phenomenon could be the sympathetic dysregulation during a migraine attack. On the other hand, the unique type of basilar-migraine attacks involving the brainstem and especially the pons with the noradrenaline-producing locus coeruleus, the serotonin-producing dorsal raphe nucleus, point more to a direct dysfunction of these structures during migraine attacks.

In our opinion, Tosun et al reported a valuable research for recognising the relationship between the autonomic nervous system disturbance and the migraine. Unfortunately, the authors did not discuss the possible contributions of the clinical subtypes of migraine and autonomic nervous system dysfunction.

\section{References}

1. Tosun O, Karatoprak E. Increased QT and P-wave dispersion during attack-free period in pediatric patients with migraine attacks. Cardiol Young 2019; 17: 1-4.

2. Matei D, Constantinescu V, Corciova C, Ignat B, Matei R, Popescu CD. Autonomic impairment in patients with migraine. Eur Rev Med Pharmacol Sci 2015; 19: 3922-3927.

3. Man YH, Meng XL, Yu TM, Yao G. Pacemaker implantation for treating migraine-like headache secondary to cardiac arrhythmia: a case report. Medicine (Baltimore) 2018; 97: e13629.

4. Monroe DJ, Meehan JT 4th, Schandl CA. Sudden cardiac death in a young man with migraine-associated arrhythmia. J Forensic Sci 2015; 60: 1633-1636.

5. Yao G, Zhang XM. A valuable case for recognizing the relationship between arrhythmia and the biology of migraine with brainstem aura. J Forensic Sci 2016; 61: 288. 